\title{
Direct Evidence for the Effect of Quantum Confinement of Surface-State Electrons on Atomic Diffusion
}

\author{
N. N. Negulyaev, ${ }^{1}$ V. S. Stepanyuk, ${ }^{2}$ L. Niebergall, ${ }^{2}$ P. Bruno, ${ }^{2,3}$ W. Hergert, ${ }^{1}$ J. Repp, ${ }^{4}$ K.-H. Rieder, ${ }^{5}$ and G. Meyer ${ }^{6}$ \\ ${ }^{1}$ Fachbereich Physik, Martin-Luther-Universität, Halle-Wittenberg, Friedemann-Bach-Platz, 6, D-06099 Halle, Germany \\ ${ }^{2}$ Max-Planck-Institut für Mikrostrukturphysik, Weinberg 2, D-06120 Halle, Germany \\ ${ }^{3}$ European Synchrotron Radiation Facility, BP 220, F-38043 Grenoble Cedex, France \\ ${ }^{4}$ Institute of Experimental and Applied Physics, University of Regensburg, D-93040 Regensburg, Germany \\ ${ }^{5}$ Institut für Experimentalphysik, Freie Universität Berlin, Arnimallee 14, D-14195 Berlin, Germany \\ ${ }^{6}$ IBM Research, Zurich Research Laboratory, CH-8803 Rüschlikon, Switzerland \\ (Received 8 July 2008; revised manuscript received 16 September 2008; published 24 November 2008)
}

\begin{abstract}
We report on the direct observations of the effect of quantum confinement of surface-state electrons on atomic diffusion. Confined electronic states induced by open nanoscale resonators [consisting of two parallel monatomic $\mathrm{Cu}$ chains on $\mathrm{Cu}(111)$ ] are studied by means of scanning tunneling microscope measurements and first-principles calculations. Strongly anisotropic diffusion of adatoms around and inside resonators is revealed at low temperatures. The formation of diffusion channels and empty zones is demonstrated. We show that it is possible to engineer atomic diffusion by varying the distance between the resonator walls.
\end{abstract}

DOI: 10.1103/PhysRevLett.101.226601

PACS numbers: 72.25. $-\mathrm{b}, 75.75 .+\mathrm{a}$

Surface-state electrons on the (111) surfaces of noble metals form a two-dimensional nearly free electron gas. During the past two decades quantum confinement of such electrons was a topic of intensive investigation. Real-space observations of ripples of conduction electrons were performed by means of scanning tunneling microscopy (STM) and scanning tunneling spectroscopy (STS) in the vicinity of surface imperfections, such as steps [1-4], adsorbates $[5,6]$, nanoscale islands $[7,8]$, subsurface defects, and vacancy holes [9].

The scattering of a 2D electron gas also gives rise to an interaction between the scatterers. This kind of interaction was predicted in 1978 by Lau and Kohn [10]. It was investigated theoretically in more detail using different techniques [11-13] before it was observed experimentally $[14,15]$. These observations strengthened the interest in this topic in the following years, in which it was used, e.g., to grow ordered structures of individual adatoms [1620]. Corresponding theory studies were extended to manybody [21,22] and spin-polarization [23] effects.

Modern progress in STM manipulation makes it possible to construct artificial nanostructures of different shapes and sizes in an atom-by-atom fashion [24]. Using such technique one can create quantum resonators for the surface-state electrons $[5,25,26]$. Since in such resonators the amplitude of surface-state standing-wave patterns can be greatly enhanced, it can be anticipated that also the interaction potential mediated by the surface-state electrons can be intensified.

In this Letter we report on the first direct observations of the effect of confined surface-state electrons on atomic diffusion. We perform combined, experimental and theoretical, study of an atomic diffusion in "handmade" quantum resonators. Using STM-manipulation technique we construct two parallel monatomic $\mathrm{Cu}$ chains on $\mathrm{Cu}(111)$ and reveal quantum confinement between them. Our $a b$ initio calculations support the confinement picture. Both experimental observations and kinetic Monte Carlo $(\mathrm{KMC})$ simulations demonstrate that diffusion of a $\mathrm{Cu}$ adatom introduced inside a resonator is strongly anisotropic: formation of diffusion channels and empty zones is revealed. We show that it is possible to control and engineer atomic diffusion on metal substrates, changing the distance between resonator walls.

Our experiments were performed in an ultrahigh vacuum chamber $\left(\approx 10^{-11}\right.$ mbar $)$, which is equipped with a lowtemperature STM operated at 9-21 K. These temperatures are low enough to record the diffusion of single $\mathrm{Cu}$ adatoms on $\mathrm{Cu}(111)$ [14]. The sample was cleaned by several sputter and anneal cycles at temperatures of about $800 \mathrm{~K}$. As STM tips we used electrochemically etched tungsten wires [27]. The calculations of the local density of states (LDOS) within resonators and the interaction between an adatom and a resonator are performed using density functional theory by means of Korringa-Kohn-Rostocker Green's function method [28]. Details of this method and its several applications can be found in the previous works $[9,17,19,23]$.

Our systematic experimental studies demonstrate that a $\mathrm{Cu}$ adatom on $\mathrm{Cu}(111)$ follows $2 \mathrm{D}$ random walk (movie 1 [29]). However, quantum resonators drastically affect atomic diffusion. First we consider a single $\mathrm{Cu}$ atom in a vicinity of a single monatomic $\mathrm{Cu}$ chain. Initially, the adatom is placed by means of STM tip at separation of about $35 \AA$ from the chain. Figure 1 (movie 2 [29]) shows the diffusion of the $\mathrm{Cu}$ adatom recorded at $T=12.5 \mathrm{~K}$ during $32 \mathrm{~h}$. One can see that the $\mathrm{Cu}$ chain dramatically influences atomic diffusion: the adatom prefers to be at 


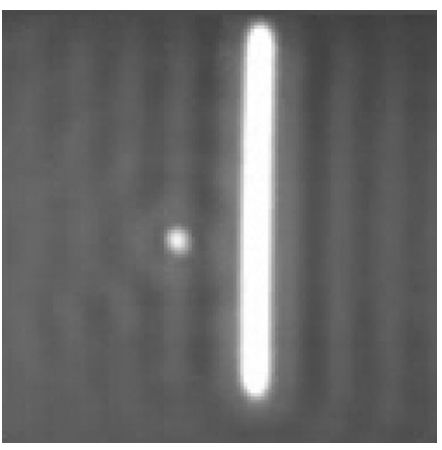

FIG. 1. (Movie 2 [29]). Diffusion of a single $\mathrm{Cu}$ adatom at a separation of about $25 \AA$ from a monatomic $\mathrm{Cu}$ chain on $\mathrm{Cu}$ (111). $T=12.5 \mathrm{~K}, I=0.5 \mathrm{nA}, V=+0.1 \mathrm{eV}$, total time $32 \mathrm{~h}$, frame size $130 \times 130 \AA$.

23-27 $\AA$ from the chain, migrating along the diffusion channel only. To explain this phenomenon, we recall our recent studies on the substrate-mediated interactions of $3 d$ adatoms with step edges on a $\mathrm{Cu}(111)$ surface [20]. Monatomic $\mathrm{Cu}$ chain can be regarded as an ascending step on $\mathrm{Cu}(111)$. The interaction between the ascending step and a $3 d$ adatom has an oscillatory behavior with an attractive minimum of $-1 \mathrm{meV}$ at separation of $25 \AA$ [see

(a)
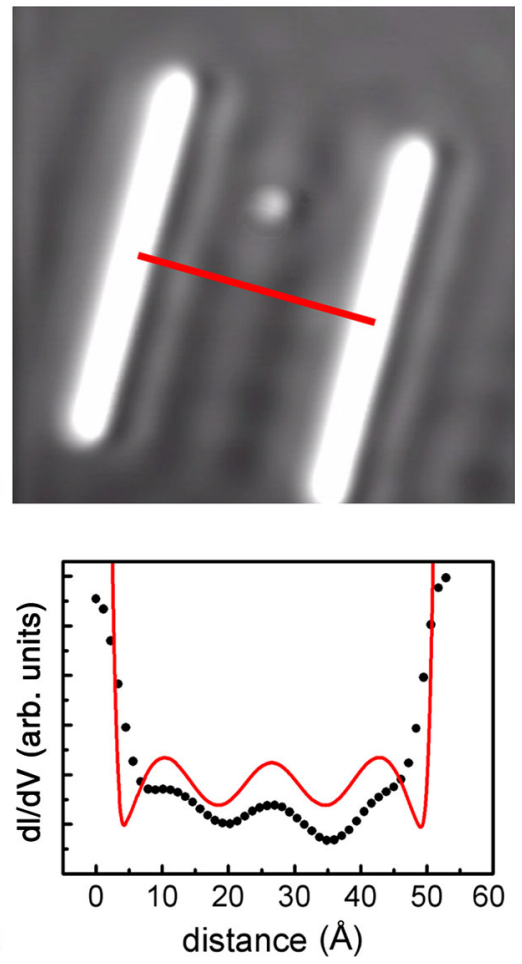

FIG. 2 (color online). (a) (Movie 3 [29]). Diffusion of a single $\mathrm{Cu}$ adatom inside a quantum resonator (two parallel monatomic $\mathrm{Cu}$ chains) on $\mathrm{Cu}(111)$. The distance between the chains is $55 \AA$. $T=12 \mathrm{~K}, I=0.07 \mathrm{nA}, V=+0.1 \mathrm{eV}$, total time $19 \mathrm{~h}$, frame size $100 \times 100 \AA$. (b) Experimentally measured $d I / d V$ signal at $V=-0.05 \mathrm{eV}$ (black dots) and the calculated LDOS at the Fermi level [gray (red) curve] perpendicular to the resonator walls [gray (red) line in (a)].
Fig. 1(a) in Ref. [20]]. This interaction energy equals the thermal energy $k_{B} T$ at $T=12 \mathrm{~K}$ and therefore is decisive for the observed diffusion behavior, resulting in the predominant diffusion parallel to the chain.

Now we turn to a single $\mathrm{Cu}$ atom inside a resonator. Figure 2(a) (see movie 3 [29]) shows the atomic diffusion recorded at $T=12 \mathrm{~K}$ during $19 \mathrm{~h}$. The distance between the $\mathrm{Cu}$ chains is $55 \AA$. The adatom is placed in a vicinity of the central line of the resonator by means of the STM tip. The observed diffusion differs from that on a flat surface: the adatom is confined in the central region and migrates parallel to the $\mathrm{Cu}$ chains only. We performed theoretical studies of quantum confinement of surface electrons within the resonator. In Fig. 2(b) we plot the cross section of the distribution of the LDOS at the Fermi energy inside the resonator perpendicular to its walls. The quantum interference between electronic waves traveling towards the $\mathrm{Cu}$ chains and the backscattered ones leads to the oscillations of the LDOS. We compare our theoretical results with the $d I / d V$ signal measured close to the Fermi level $(V=$ $-0.05 \mathrm{eV}$ ). The calculations reproduce the oscillations of the LDOS found experimentally: there is a perfect agreement in the number of peaks and their positions.

The relative position of the LDOS and the interaction potential depends on the Fermi-level-phase shift [11]. A surface location with a high LDOS constitutes a preferred binding site for an adatom $[1,14,16]$. Figure 3 presents the 2D potential energy map of the $\mathrm{Cu}$ adatom placed in different hollow sites between $\mathrm{Cu}$ chains. The interaction potential exhibits an oscillatory behavior if one recedes from a $\mathrm{Cu}$ chain towards the central line of the resonator. When the adatom is located at the first nearest neighbor separation from a chain, the interaction is strongly attrac-

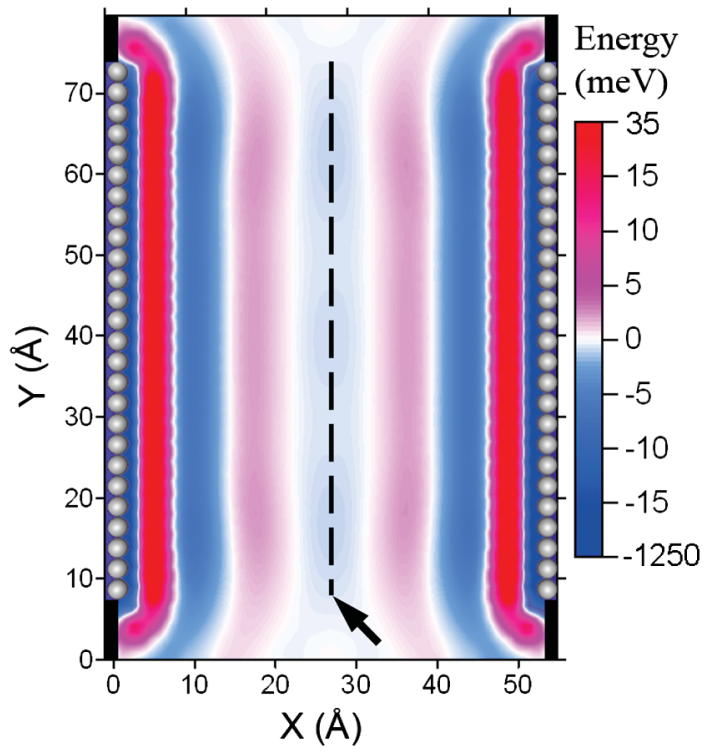

FIG. 3 (color). The 2D map of the interaction potential between a $\mathrm{Cu}$ adatom and the quantum resonator (two parallel monatomic $\mathrm{Cu}$ chains with interchain separation of $55 \AA$ ). The black dashed line marks the central line of the resonator. 
tive being $-1.25 \mathrm{eV}$. There is a repulsive barrier of $33 \mathrm{meV}$ at $4-9 \AA$ from the chain; it prevents nucleation of the adatom and a chain at low temperatures. It is energetically favorable for the $\mathrm{Cu}$ adatom to be located at 9-15 $\AA$ (the minimum is $-6 \mathrm{meV}$ ). The adatom localized at such distances is confined between the repulsive barriers that arose at 4-9 $\AA$ and 16-23 $\AA$ (the last one has magnitude of $2 \mathrm{meV}$ ). There is yet another minimum of $-1 \mathrm{meV}$ along the central line of the resonator.

In order to quantify the effect of the quantum confinement on atomic diffusion theoretically, we perform KMC study $[12,13,30,31]$ of behavior of a $\mathrm{Cu}$ adatom placed between two $\mathrm{Cu}$ chains (see details in Ref. [20]). The adatom probability distribution inside the resonator is shown in Fig. 4(a). Red and violet colors mark diffusion channels, i.e., regions where an adatom is localized with a high probability. They correspond to the areas of negative interaction energy (Fig. 3). Strong modulation of the probability distribution inside the resonator with respect to that on a flat surface [Fig. 4(b)] is well seen.

Three diffusion channels exist [Fig. 4(a)]. An adatom located within any of these channels diffuses predominantly parallel to the resonator walls. When the adatom is introduced in the region of a low LDOS (between the channels), first it migrates towards one of the neighboring channels, i.e., perpendicular to the resonator walls.

One of the most exciting effects is "trapping" the adatom: the adatom tends to stay within the resonator, despite

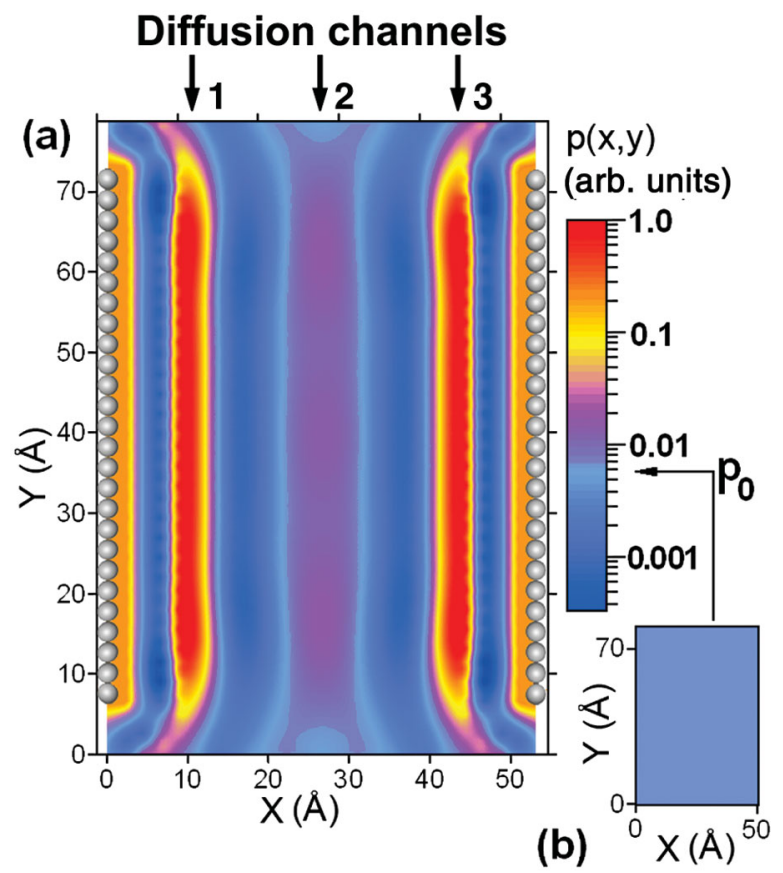

FIG. 4 (color). (a) Atomic diffusion inside the quantum resonator from Fig. 2(a): results of the KMC simulations for a single $\mathrm{Cu}$ adatom. The probability to find a randomly introduced $\mathrm{Cu}$ atom is calculated at $12 \mathrm{~K}$. (b) The corresponding probability $p_{0}$ to find a randomly introduced $\mathrm{Cu}$ atom at the absence of the quantum resonator. the fact that the nanostructure is open (movie 3). This phenomenon can be understood if we follow the interaction potential along the central line of the resonator (Fig. 3). The energy is attractive (about $-1 \mathrm{meV}$ ) if the adatom is between the chains, while it is zero when the adatom is outside the resonator. However, we note that all diffusion channels [Fig. 4(a)] are open: there is always a certain probability that the adatom escapes from the resonator. The diffusion time of the adatom inside the channel is proportional to $L^{2}$ (where $L$ is the length of the resonator) and $\exp \left(-E / k_{B} T\right)$ (where $E$ is the interaction energy "adatom resonator" within the channel at the edge of the resonator (see the black arrow in the Fig. 3).

Since the period of oscillations of standing-wave patterns depends on the Fermi wavelength $\lambda_{F}$ of the surface only $[1,11]$, one can tune the number of diffusion channels varying the distance between resonator walls. To confirm this statement we study atomic diffusion within a smaller resonator (interchain distance is $35 \AA$ ). Two $\mathrm{Cu}$ adatoms are introduced between $\mathrm{Cu}$ chains [32], and their diffusion is examined at $T=13 \mathrm{~K}$ during $30 \mathrm{~h}$ (Fig. 5, movie 4 [29]). The adatoms demonstrate correlated motion parallel to the resonator walls. Finally, one of the $\mathrm{Cu}$ adatoms escapes: it turns around the edge atom of the chain.

To reveal the physics behind this experiment, first we plot the $2 \mathrm{D}$ potential energy map of a single $\mathrm{Cu}$ adatom placed between $\mathrm{Cu}$ chains [Fig. 6(a)]. There are two stripes of attractive interaction, confined between the regions of repulsive energies. Diffusion channels (empty zones) correspond to an attractive (repulsive) interaction between a $\mathrm{Cu}$ adatom and the resonator. Two diffusion channels exist, and both $\mathrm{Cu}$ adatoms migrate inside the resonator within these channels only (movie 4). To understand the correlated atomic motion we employ KMC study [12,13,30,31] of behavior of two $\mathrm{Cu}$ adatoms introduced between two $\mathrm{Cu}$ chains. The long-range interaction (LRI) between two $\mathrm{Cu}$ adatoms on $\mathrm{Cu}(111)$ is taken from Ref. [33]. In Fig. 6(b) we plot the distribution of the interatomic distance between

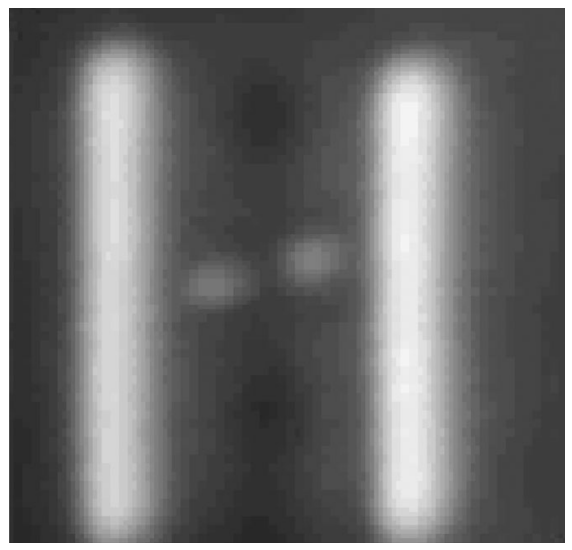

FIG. 5. (Movie 4 [29]). Diffusion of two $\mathrm{Cu}$ atoms inside quantum resonator (two parallel monatomic $\mathrm{Cu}$ chains) on $\mathrm{Cu}$ (111). The distance between the chains is $35 \AA . T=13 \mathrm{~K}, I=$ $0.2 \mathrm{nA}, V=+0.1 \mathrm{eV}$, total time $30 \mathrm{~h}$, frame size $65 \times 65 \AA$. 

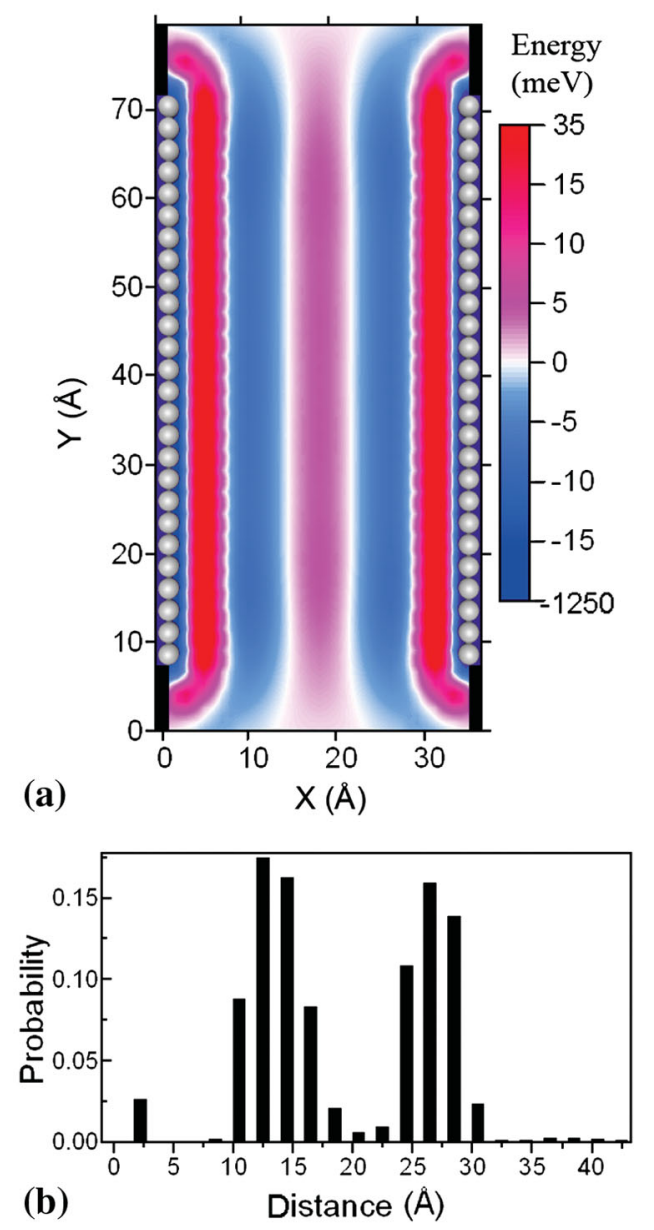

FIG. 6 (color). (a) The 2D map of the interaction potential between a $\mathrm{Cu}$ adatom and the quantum resonator (two parallel $\mathrm{Cu}$ chains, interchain separation of $35 \AA$ ). (b) Distribution of the $\mathrm{Cu}-\mathrm{Cu}$ interatomic distance during atomic diffusion (movie 4): the result of the KMC simulations.

two $\mathrm{Cu}$ adatoms. This function has two pronounced maxima at 13 and $26 \AA$, which are caused by two minima (at 11 and $26 \AA$ ) of the LRI potential between two $\mathrm{Cu}$ adatoms on $\mathrm{Cu}(111)$ [33]. From Fig. 6(b) we conclude that the $\mathrm{Cu}$ atoms form a pair stabilized by the LRI. Our calculations demonstrate that the probability for an adatom in the pair to leave the resonator shown in Fig. 5 is by $25 \%$ less than in the case of a single adatom.

In summary, we have reported on the direct observations of the effect of confined surface-state electrons on atomic diffusion. A combined experimental and theoretical investigation of the quantum confinement of conduction electrons around and inside nanoscale resonators has been performed. We have unambiguously demonstrated that standing-wave patterns of electronic states significantly modify the atomic diffusion at low temperatures and that it is possible to control and engineer the atomic diffusion varying the distance between resonator walls.

This work was supported by Deutsche Forschungsgemeinschaft (SPP1165 and SPP1153). J.R. gratefully acknowledges funding by the Volkswagen Foundation through its Lichtenberg program.

[1] M.F. Crommie, C.P. Lutz, and D. M. Eigler, Nature (London) 363, 524 (1993).

[2] Y. Hasegawa and Ph. Avouris, Phys. Rev. Lett. 71, 1071 (1993).

[3] J. M. Garcia et al., Appl. Phys. A 61, 609 (1995).

[4] L. Bürgi et al., Phys. Rev. Lett. 81, 5370 (1998); M. Pivetta et al., Phys. Rev. B 67, 193402 (2003).

[5] M. F. Crommie, C. P. Lutz, and D. M. Eigler, Science 262, 218 (1993).

[6] Ph. Avouris et al., J. Vac. Sci. Technol. B 12, 1447 (1994).

[7] J. Li et al., Phys. Rev. Lett. 80, 3332 (1998).

[8] L. Diekhöner et al., Phys. Rev. Lett. 90, 236801 (2003).

[9] G. Rodary et al., Phys. Rev. B 75, 233412 (2007).

[10] H. Lau and W. Kohn, Surf. Sci. 75, 69 (1978).

[11] P. Hyldgaard and M. Persson, J. Phys. Condens. Matter 12, L13 (2000).

[12] K. A. Fichthorn and M. Scheffler, Phys. Rev. Lett. 84, 5371 (2000).

[13] A. Bogicevic et al., Phys. Rev. Lett. 85, 1910 (2000).

[14] J. Repp et al., Phys. Rev. Lett. 85, 2981 (2000).

[15] N. Knorr et al., Phys. Rev. B 65, 115420 (2002).

[16] F. Silly et al., Phys. Rev. Lett. 92, 016101 (2004); F. Silly et al., New J. Phys. 6, 16 (2004).

[17] V. S. Stepanyuk et al., Phys. Rev. B 68, 205410 (2003).

[18] N. N. Negulyaev et al., Surf. Sci. 600, 58 (2006); N. N. Negulyaev et al., Phys. Rev. B 74, 035421 (2006).

[19] V. S. Stepanyuk et al., Phys. Rev. Lett. 97, 186403 (2006).

[20] H. F. Ding et al., Phys. Rev. B 76, 033409 (2007).

[21] P. Hyldgaard and T. L. Einstein, Europhys. Lett. 59, 265 (2002); Surf. Sci. 532, 600 (2003).

[22] K. A. Fichthorn, M. L. Merrick, and M. Scheffler, Phys. Rev. B 68, 041404 (2003).

[23] V. S. Stepanyuk et al., Phys. Rev. Lett. 94, 187201 (2005); L. Niebergall et al., Phys. Rev. Lett. 96, 127204 (2006).

[24] D. M. Eigler and E. K. Schweizer, Nature (London) 344, 524 (1990).

[25] H. C. Manoharan, C.P. Lutz, and D. M. Eigler, Nature (London) 403, 512 (2000).

[26] K.-F. Braun and K.-H. Rieder, Phys. Rev. Lett. 88, 096801 (2002).

[27] In all measurements bias voltage refers to the sample voltage with respect to the tip.

[28] K. Wildberger et al., Phys. Rev. Lett. 75, 509 (1995); V. S. Stepanyuk et al., Phys. Rev. B 53, 2121 (1996).

[29] See EPAPS Document No. E-PRLTAO-101-017849 for diffusion of a $\mathrm{Cu}$ adatom on a flat surface (movie 1), in a vicinity of the monatomic $\mathrm{Cu}$ chain (movie 2), inside the quantum resonator (movie 3), and correlated diffusion of two $\mathrm{Cu}$ adatoms inside the quantum resonator (movie 4). For more information on EPAPS, see http://www.aip.org/ pubservs/epaps.html.

[30] K. A. Fichthorn and W. H. Weinberg, J. Chem. Phys. 95, 1090 (1991).

[31] S. Ovesson et al., Phys. Rev. B 64, 125423 (2001).

[32] The first $\mathrm{Cu}$ atom was introduced in the vicinity of the left wall of the resonator, while another one was introduced near the right one.

[33] V. S. Stepanyuk et al., Comput. Mater. Sci. 35, 272 (2006). 